\title{
CARGA SEMANAL DE TRABALHO PARA ENFERMEIROS NO BRASIL: DESAFIOS AO EXERCÍCIO DA PROFISSÃO
}

\author{
WEEKLY WORKLOAD FOR NURSES IN BRAZIL: CHALLENGES \\ TO PRACTICE THE PROFESSION \\ CARGA SEMANAL DE TRABAJO PARA ENFERMEROS EN BRASIL: \\ DESAFÍOS PARA EL EJERCICIO DE LA PROFESIÓN
}

Bruno Luciano Carneiro Alves de Oliveira ${ }^{1}$

\author{
Alécia Maria da Silva ${ }^{2}$ \\ Sara Fiterman Lima $^{3}$
}

Resumo Este estudo teve por objetivo apresentar a distribuição de enfermeiros no mercado de trabalho com carga horária semanal maior ou igual a quarenta horas segundo as regiões e o local público ou privado de trabalho no Brasil. Tratou-se de estudo transversal, realizado entre 10 e 27 de fevereiro de 2017, com dados on-line das pesquisas de assistência médico-sanitária realizadas em 2002, 2005 e 2009 pelo Instituto Brasileiro de Geografia e Estatística. Analisou-se um total de 179.337 enfermeiros nos três anos avaliados. Compararam-se as suas proporções de acordo com as regiões geográficas e o local de trabalho utilizando-se o teste qui-quadrado de Pearson $(a=5 \%)$. A enfermagem foi a única das profissões da saúde de nível superior que apresentou crescente e maior proporção de profissionais com carga de trabalho maior ou igual a quarenta horas semanais $(p<0,001)$ nos anos avaliados, em que a maior proporção é no setor público e nas regiões Norte, Centro-Oeste e Sul do país. A enfermagem ainda permanece com carga de trabalho desfavorável, que consiste em desafio para os enfermeiros e para a enfermagem como profissão.

Palavras-chave enfermagem; saúde e desigualdade; mercado de trabalho.
Abstract The study had the goal of presenting the distribution of nurses in the job market with a weekly workload greater than or equal to forty hours according to the regions of Brazil and the kind of workplace (whether in the private or public sectors). It was a cross-sectional study, conducted between 10 and 27 of February 2017, with on-line data from the medical-sanitary researches conducted in 2002, 2005 and 2009 by the Brazilian Institute of Geography and Statistics. We analyzed a total of 179,337 nurses in the 3 evaluated years. We compared their proportions according to each geographical region of Brazil and the workplace using Pearson's Chi-squared test $(a=5 \%)$. Nursing was the only college-level healthcare occupation that presented a larger and increasing proportion of professionals with a weekly workload greater than or equal to 40 hours ( $\mathrm{p}<0.001)$, and this proportion is greater in the public sector and in the Northern, Midwestern, and Southern regions of the country. The occupation of nurse still has an unfavorable workload, which constitutes a challenge to the nurses and to nursing as a profession.

Keywords nursing; health and inequality; job market. 


\section{Introdução}

Atualmente, a enfermagem é a profissão mais frequente entre todas as que compõem o mercado de trabalho do setor saúde brasileiro (Oliveira, Silva e Carneiro, 2015). Na última década, o número e a proporção de enfermeiros no Brasil inseridos no mercado de trabalho desse setor cresceram progressivamente, sendo de 12,2\% (88.952) em 2002 (Instituto Brasileiro de Geografia e Estatística, 2002), aumentando para 13,3\% (116.126) em 2005 (Instituto Brasileiro de Geografia e Estatística, 2006) e para 14,8\% (163.099) em 2009 (Instituto Brasileiro de Geografia e Estatística, 2010). Além disso, a enfermagem faz parte das profissões essenciais, representando uma das 14 profissões do setor saúde, e apresenta estruturante papel no sistema de saúde, pois está presente em quase todas as instituições que prestam assistência à saúde e desempenha um trabalho essencial no cuidado à vida humana (Bogaert et al., 2013; Oliveira, Silva e Carneiro, 2015; Silva Neto et al., 2015).

Embora a enfermagem seja amplamente reconhecida como atividade pública de inquestionável valor social, científico e tecnológico; tenha influência decisiva na qualidade das ações e serviços de saúde; venha se consolidando como campo de conhecimento e práticas específicas, de atenção à saúde da população; e apresente-se com grande contingente numérico de profissionais, os enfermeiros ainda têm carga horária de trabalho semanal excessiva e desproporcional à de outros profissionais da saúde de nível superior no Brasil. Essa carga horária de trabalho revela-se como uma das principais consequências negativas do modelo político-hegemônico capitalista que se expressa institucionalmente no contexto do trabalho globalizado (Pires et al., 2010; Dalri et al., 2014a; Oliveira, Silva e Carneiro, 2015).

Em vários países, trabalhos mostram que a enfermagem tem longas jornadas de trabalho, associadas a más condições laborais, pouca autonomia, muita responsabilidade e baixa valorização, que geram elevada demanda física e emocional (Pires et al., 2010; Gray-Stanley e Muramatsu, 2011; Bogaert et al., 2013; Dalri et al., 2014a; Silva Neto et al., 2015; Lala et al., 2016). Tais situações têm contribuído para a perda parcial ou total das capacidades corporais e psíquicas desses profissionais, com prejuízos na vida cotidiana em razão da exaustão física e da emocional, que se apresentam de modo mais frequente em enfermeiros do que entre a população em geral (Gray-Stanley e Muramatsu, 2011; Bogaert et al., 2013; Lala et al., 2016).

Os trabalhadores de enfermagem geralmente possuem vários vínculos de trabalho, que se caracterizam por serem de baixos salários. Costumam assumir dupla jornada de trabalho em turnos diferentes, como forma de compensar os baixos rendimentos (Santos et al., 2013). Dessa forma, a enfermagem tem função ininterrupta, associada aos múltiplos vínculos, que exigem muita atenção e resultam em impactos na qualidade e segurança do cuidado, na satisfa- 
ção profissional e no reconhecimento social (Bogaert et al., 2013; Lima et al., 2013; Dalri et al., 2014a; Lala et al., 2016). Esses fatores desafiam o exercício da enfermagem nos diferentes contextos socioeconômicos e de setores de trabalho no Brasil (Oliveira, Silva e Carneiro, 2015; Silva Neto et al., 2015). Além disso, ao contrário de outras categorias profissionais da saúde, que já obtiveram conquistas em âmbito nacional em relação ao controle e redução da jornada de trabalho, a enfermagem vem em processo de luta e de embates importantes na tentativa de instituir nacionalmente a proteção legal ao seu trabalho em torno de uma carga horária mais favorável e de qualidade de vida laboral (Pires et al., 2010; Dalri et al., 2014a, 2014b).

O processo de luta histórica pela regulamentação de uma jornada de trabalho compatível com as necessidades do trabalho e de profissionais da enfermagem é bastante antigo e já ultrapassa meio século. Na lei n. 2.604/1955, que regulamentou o exercício profissional da enfermagem, o único veto foi no artigo que estabelecia a jornada máxima de trinta horas semanais (Pires et al., 2010; Lima et al., 2013; Santos et al., 2013). No início dos anos 1980, em 1983, um projeto de lei (n. 3.225-c/80) foi aprovado no Congresso Nacional e vetado pelo então presidente João Baptista Figueiredo. Este projeto fixava piso salarial de dez salários mínimos para trinta horas semanais para o enfermeiro. Em 1989, dando continuidade à luta, foi encaminhado ao Congresso Nacional novo projeto de lei (n. 4.499/89). Após longo processo de negociação, desvinculou-se do projeto a questão do piso salarial, para permitir tramitação no Congresso Nacional. Porém, em 1993, o novo projeto deu entrada, tramitou e foi novamente aprovada a regulamentação da jornada de trabalho em trinta horas, com a aprovação do projeto de lei n. 407/1991 na Câmara dos Deputados e no Senado, mas foi novamente vetado pelo então presidente da República, Fernando Henrique Cardoso, em 21/12/1996.

Mais recentemente, o Conselho Federal de Enfermagem (Cofen), por meio da resolução n. 293/2004, regulamentou jornada de 36 horas semanais para atividade assistencial e de quarenta horas semanais para atividades administrativas. O processo de luta da profissão por uma jornada máxima de trinta horas tem se mobilizado nos últimos vinte anos em torno do projeto de lei n. 2.295/2000 (Pires et al., 2010; Santos et al., 2013; Dalri et al., 2014a; Silva Neto et al., 2015).

A importância e a necessidade dessa reivindicação já foram inclusive reconhecidas em conferências de saúde, no Legislativo, no Judiciário, pelos meios de comunicação e pelas entidades que representam profissionais e usuários dos serviços de saúde. Entretanto, essa conquista tem sido postergada em razão de interesses econômicos e capitalistas (Pires et al., 2010; Santos et al., 2013; Dalri et al., 2014a; Silva Neto et al., 2015).

$\mathrm{O}$ reconhecimento também vem sendo legalmente instituído em âmbitos local e estadual, mas ainda não beneficia todos os enfermeiros do país, não 
representando uma conquista coletiva, uniforme e comum em um país com tantas disparidades socioeconômicas, regionais e de tipos de serviços de saúde (Pires et al., 2010; Dalri et al., 2014a, 2014b).

Portanto, a partir dessas reflexões, objetivou-se apresentar a distribuição de enfermeiros inseridos no mercado de trabalho do setor saúde com carga horária semanal maior ou igual a quarenta horas no trabalho principal segundo as macrorregiões geográficas e o local de trabalho, público ou privado, no Brasil nos anos de 2002, 2005 e 2009.

\section{As Pesquisas de Assistência Médico-Sanitária}

O estudo aqui apresentado foi do tipo transversal, realizado entre 10 e $27 \mathrm{de}$ fevereiro de 2017, baseado nos dados on-line das pesquisas de assistência médico-sanitária (PAMSs) realizadas nos anos de 2002, 2005 e 2009. A PAMS é uma pesquisa censitária de abrangência nacional que foi realizada regularmente pela Fundação Instituto Brasileiro de Geografia e Estatística (IBGE) em parceria com o Ministério da Saúde do Brasil até 2009. Desde então, o IBGE ainda não tem disponibilizado dados mais recentes sobre todos os estabelecimentos de saúde existentes no país (Instituto Brasileiro de Geografia e Estatística, (2010).

A PAMS foi implementada com o objetivo de revelar de modo mais preciso o perfil das tendências de oferta de serviços, profissionais de saúde e da capacidade instalada dos serviços de saúde no Brasil. A pesquisa é um instrumento valioso e que disponibiliza informações sobre os estabelecimentos e os profissionais de saúde existentes no país (Instituto Brasileiro de Geografia e Estatística, 2002, 2006, 2010). Este censo é realizado por meio de entrevistas e abrange todos os estabelecimentos de saúde existentes no país que prestam assistência à saúde individual ou coletiva, com um mínimo de técnica apropriada, segundo critérios estabelecidos pelo próprio Ministério da Saúde, para atendimento rotineiro à população, em serviços públicos ou privados, com ou sem fins lucrativos, em regime ambulatorial ou de internação. Também são incluídos estabelecimentos que realizam exclusivamente serviços de apoio à diagnose, terapia e controle regular de zoonoses (Instituto Brasileiro de Geografia e Estatística, 2002, 2006, 2010).

As informações dos estabelecimentos foram coletadas em questionários de acordo com o tipo de complexidade dos serviços oferecidos. Também se levantou a situação dos postos de trabalho dos profissionais da saúde inseridos nesses estabelecimentos. Nas PAMSs, foram relacionadas informações de 729.746, 870.361 e 1.104.340 profissionais da saúde em 2002, 2005 e 2009, respectivamente. Para o estudo aqui apresentado, selecionaram-se dados referentes aos enfermeiros que participaram da PAMS nos anos mencionados e que tinham carga de trabalho semanal maior ou igual a quarenta horas, o que 
permitiu a análise de 179.337 enfermeiros, assim distribuídos: 39.892 participantes da pesquisa de 2002; 57.159 da pesquisa de 2005; e 82.286 da pesquisa de 2009 (Instituto Brasileiro de Geografia e Estatística, 2002, 2006, 2010).

A distribuição dos enfermeiros avaliados no estudo foi apresentada por meio de frequências absolutas e relativas. Estimaram-se as prevalências para enfermeiros no Brasil, nas cinco regiões geográficas do país e nos postos de trabalho público (nos âmbitos federal, estadual e municipal) e privado segundo a condição de trabalho $\geq 40$ horas. Essas estimativas foram feitas, respectivamente, em relação ao total de enfermeiros em 2002, 2005 e 2009.

Para comparar se houve diferenças nas prevalências de enfermeiros, segundo os critérios de interesse nos anos estudados, utilizou-se o teste quiquadrado de Pearson com nível de significância em 5\%. As análises foram feitas no software Stata versão 14.0 (Stata Corp., College Station, Texas, EUA).

Os dados secundários utilizados no estudo são de domínio público, e não há até o presente momento a obrigação de solicitação prévia a instituições ou órgãos governamentais para seu manejo e publicação. Por isso não foi submetido a Comitê de Ética em Pesquisa.

\section{Resultados}

O estudo incluiu 179.337 enfermeiros identificados nas PAMSs realizadas pelo IBGE nos anos de 2002, 2005 e 2009, assim distribuídos: 39.892 participantes da pesquisa de 2002, 57.159 da pesquisa de 2005 e 82.286 da pesquisa de 2009 (Tabela 1).

\section{Tabela 1}

\begin{tabular}{|c|c|c|c|c|c|c|c|c|c|}
\hline \multicolumn{10}{|c|}{$\begin{array}{l}\text { Distribuição absoluta e relativa dos profissionais de nível superior no Brasil com carga horária semanal de } \\
\text { trabalho no emprego principal } \geq 40 \text { horas e o ano de pesquisa (PAMS 2002, 2005, 2009) }\end{array}$} \\
\hline & \multicolumn{2}{|c|}{2002} & \multicolumn{2}{|c|}{2005} & \multicolumn{2}{|c|}{2009} & \multirow{2}{*}{$\begin{array}{c}p \text {-valor } \\
2002, \\
2005 \text { e } \\
2009\end{array}$} & \multirow{2}{*}{$\begin{array}{c}p \text {-valor* } \\
2005 \text { vs } \\
2002\end{array}$} & \multirow{2}{*}{$\begin{array}{c}p \text {-valor } \\
2009 \text { vs } \\
2002\end{array}$} \\
\hline & $\mathbf{n}$ & $\%$ & $\mathbf{n}$ & $\%$ & $\mathbf{n}$ & $\%$ & & & \\
\hline Médicos & 92.577 & 19,9 & 115.302 & 21,9 & 142.900 & 22,5 & 0,001 & 0,001 & 0,001 \\
\hline Odontólogos & 10.878 & 19,1 & 20.743 & 29,1 & 29.840 & 31,7 & 0,001 & 0,001 & 0,001 \\
\hline Enfermeiros & 39.892 & 44,8 & 57.159 & 49,2 & 82.286 & 50,5 & 0,001 & 0,001 & 0,001 \\
\hline Outros & 39.918 & 33,9 & 51.463 & 33,2 & 73.361 & 41,4 & 0,001 & 0,001 & 0,001 \\
\hline
\end{tabular}

Fonte: Instituto Brasileiro de Geografia e Estatística, 2002, 2006, 2010.

*Teste qui-quadrado de Pearson.

Embora tenham aumentado significativamente $(\mathrm{p}<0,001)$ as proporções de profissionais de saúde com carga horária de trabalho $\geq 40$ horas semanais no emprego principal, a enfermagem foi a única dessas profissões de nível 
superior nos três anos avaliados que apresentou $(\mathrm{p}<0,001)$ a maioria de seus profissionais com essa carga horária ( $\geq 40$ horas). Essas proporções aumentaram de 44,8\%, em 2002, para 49,2\%, em 2005, e atingiram 50,5\% em 2009. Em comparação aos médicos e odontólogos, essas proporções foram cerca de 2,5 vezes maiores (Tabela 1 ).

Cresceu significativamente $(\mathrm{p}<0,001)$ ao longo dos anos avaliados a proporção de enfermeiros que trabalham $\geq 40$ horas no emprego principal em todas as regiões do Brasil, e em todos os anos e regiões essas proporções foram maiores do que 40,8\%. Os maiores percentuais ocorreram nas regiões Norte, Centro-Oeste e Sul, alcançando nessas regiões estimativas $\geq 55,3 \%$ em 2009 ; e menores no Nordeste e no Sudeste, com valores até $50,8 \%$ nos anos avaliados $(\mathrm{p}<0,001)$ (Tabela 2).

Tabela 2

Distribuição absoluta e relativa de enfermeiros no Brasil com carga horária semanal de trabalho no emprego principal de $\geq 40$ horas segundo as regiões geográficas e o ano de pesquisa (PAMS, 2002, 2005, 2009)

\begin{tabular}{|c|c|c|c|c|c|c|c|c|c|}
\hline & \multicolumn{2}{|c|}{2002} & \multicolumn{2}{|c|}{2005} & \multicolumn{2}{|c|}{2009} & \multirow{2}{*}{$\begin{array}{c}\text { p-valor* } \\
2002 \\
2005 \text { e } \\
2009\end{array}$} & \multirow{2}{*}{$\begin{array}{c}p \text {-valor* } \\
2005 \text { vs } \\
2002\end{array}$} & \multirow{2}{*}{$\begin{array}{c}p \text {-valor* } \\
2009 \text { vs } \\
2002\end{array}$} \\
\hline & $\begin{array}{c}n \\
(39.892)\end{array}$ & $\%$ & $\begin{array}{c}n \\
(57.159)\end{array}$ & $\%$ & $\begin{array}{c}n \\
(82.286)\end{array}$ & $\%$ & & & \\
\hline Norte & 2.412 & 49,4 & 3.759 & 55,0 & 5.696 & 55,3 & 0,001 & 0,001 & 0,001 \\
\hline Nordeste & 10.416 & 44,8 & 16.223 & 51,5 & 17.757 & 41,4 & 0,001 & 0,001 & 0,001 \\
\hline Centro-Oeste & 2.614 & 49,9 & 4.082 & 58,4 & 6.233 & 63,0 & 0,001 & 0,001 & 0,001 \\
\hline Sudeste & 17.441 & 40,8 & 23.797 & 44,0 & 39.497 & 50,8 & 0,001 & 0,001 & 0,001 \\
\hline Sul & 7.009 & 54,5 & 9.298 & 55,4 & 13.103 & 59,2 & 0,001 & 0,129 & 0,001 \\
\hline
\end{tabular}

Fonte: Instituto Brasileiro de Geografia e Estatística, 2002, 2006, 2010.

*Teste qui-quadrado de Pearson.

Quando analisada essa distribuição segundo o local de trabalho público ou privado, observou-se que nos três anos avaliados essas proporções foram elevadas e aumentaram em ambos os setores, mas permaneceram maiores as proporções nos serviços públicos de saúde do Brasil com percentuais sempre a partir de $65 \%$ em todos os anos de análise $(\mathrm{p}<0,001)$. Entre as regiões do país também se observaram maiores percentuais no setor público, e essas proporções cresceram entre os anos avaliados, sendo maiores nas regiões Norte, Centro-Oeste e Sul, com percentuais sempre $\geq 50,7 \%$, e menores no Nordeste e no Sudeste, com percentuais de até $50,2 \%$. Já no setor privado, a proporção de enfermeiros com $\geq 40$ horas semanais no emprego principal entre os dez anos de análise aumentou nas regiões Centro-Oeste, Sudeste e Sul, e diminuiu nas regiões Norte e Nordeste $(\mathrm{p}<0,001)$ (Tabela 3). 
Tabela 3

Distribuição absoluta e relativa de enfermeiros no Brasil com carga horária semanal de trabalho no emprego principal $\geq 40$ horas segundo os postos de trabalho público ou privado entre as regiões geográficas e o ano de pesquisa (PAMS, 2002, 2005, 2009)

\begin{tabular}{|c|c|c|c|c|c|c|c|c|c|}
\hline \multirow[b]{3}{*}{ Público (Brasil) } & \multicolumn{2}{|c|}{2002} & \multicolumn{2}{|c|}{2005} & \multicolumn{2}{|c|}{2009} & \multirow{2}{*}{$\begin{array}{c}p \text {-valor* } \\
2002 \\
2005 \text { e } \\
2009\end{array}$} & \multirow{2}{*}{$\begin{array}{c}p \text {-valor* } \\
2005 \text { vs } \\
2002\end{array}$} & \multirow{2}{*}{$\begin{array}{c}p \text {-valor* } \\
2009 \text { vs } \\
2002\end{array}$} \\
\hline & $\begin{array}{c}n \\
(39.892)\end{array}$ & $\%$ & $\begin{array}{c}n \\
(57.159)\end{array}$ & $\%$ & $\begin{array}{c}n \\
(82.286)\end{array}$ & $\%$ & & & \\
\hline & 25.945 & 65,0 & 38.941 & 68,1 & 55.772 & 67,8 & 0,001 & 0,001 & 0,001 \\
\hline Norte & 2.081 & 50,7 & 3.469 & 57,4 & 5.208 & 58,3 & 0,001 & 0,001 & 0,001 \\
\hline Nordeste & 7.934 & 46,0 & 12.103 & 50,6 & 14.288 & 41,9 & 0,001 & 0,001 & 0,001 \\
\hline Centro-Oeste & 1.929 & 50,9 & 3.127 & 59,8 & 4.725 & 64,0 & 0,001 & 0,001 & 0,001 \\
\hline Sudeste & 9.517 & 37,4 & 14.083 & 42,8 & 23.368 & 50,2 & 0,001 & 0,001 & 0,001 \\
\hline Sul & 4.484 & 61,3 & 6.159 & 63,1 & 8.183 & 63,6 & 0,001 & 0,001 & 0,001 \\
\hline Privado (Brasil) & 13.947 & 44,9 & 18.218 & 47,6 & 26.514 & 49,8 & 0,001 & 0,001 & 0,001 \\
\hline Norte & 331 & 43,5 & 290 & 36,3 & 488 & 35,6 & 0,001 & 0,001 & 0,001 \\
\hline Nordeste & 2.482 & 41,1 & 4.120 & 54,4 & 3.469 & 39,3 & 0,001 & 0,001 & 0,001 \\
\hline Centro-Oeste & 685 & 47,4 & 955 & 54,5 & 1.508 & 60,1 & 0,001 & 0,343 & 0,001 \\
\hline Sudeste & 7.924 & 45,8 & 9.714 & 46,0 & 16.129 & 51,6 & 0,001 & 0,001 & 0,001 \\
\hline Sul & 2.525 & 45,5 & 3.139 & 44,7 & 4.920 & 53,0 & 0,001 & 0,042 & 0,001 \\
\hline
\end{tabular}

Fonte: Instituto Brasileiro de Geografia e Estatística, 2002, 2006, 2010.

*Teste qui-quadrado de Pearson.

Ao se analisar a distribuição dos enfermeiros no serviço público, observou-se que esses percentuais aumentaram nos três tipos de serviços públicos estudados (federal, estadual e municipal) ao longo dos anos de análise. A maioria dos enfermeiros inseridos em serviços de âmbitos federal e municipal tem carga horária de trabalho semanal $\geq 40$ horas no emprego principal. Entre 2002 e 2009, essas proporções aumentaram significativamente de $57,6 \%$ para $64,2 \%$ no âmbito federal e de $49,6 \%$ para $56,2 \%$ no âmbito municipal ( $<<0,001$ ) (Tabela 4 ).

\section{Tabela 4}

Distribuição absoluta e relativa de enfermeiros no Brasil com carga horária semanal de trabalho no emprego principal $\geq 40$ horas segundo os postos de trabalho público, federal, estadual e municipal, entre as regiões geográficas e o ano de pesquisa (PAMS, 2002, 2005, 2009)

\begin{tabular}{|c|c|c|c|c|c|c|c|c|c|}
\hline \multirow[b]{3}{*}{ Federal (Brasil) } & \multicolumn{2}{|c|}{2002} & \multicolumn{2}{|c|}{2005} & \multicolumn{2}{|c|}{2009} & \multirow{2}{*}{$\begin{array}{c}p \text {-valor* } \\
2002 \\
2005 \text { e } \\
2009\end{array}$} & \multirow{2}{*}{$\begin{array}{c}p \text {-valor* } \\
2005 \text { vs } \\
2002\end{array}$} & \multirow{2}{*}{$\begin{array}{c}p \text {-valor* } \\
2009 \text { vs } \\
2002\end{array}$} \\
\hline & $\begin{array}{c}n \\
(25.945)\end{array}$ & $\%$ & $\begin{array}{c}n \\
(38.941)\end{array}$ & $\%$ & $\begin{array}{c}n \\
(55.772)\end{array}$ & $\%$ & & & \\
\hline & 3.174 & 57,6 & 4.033 & 61,3 & 5.668 & 64,2 & 0,001 & 0,001 & 0,001 \\
\hline Norte & 134 & 53,6 & 134 & 43,9 & 240 & 35,6 & 0,049 & 0,045 & 0,978 \\
\hline Nordeste & 683 & 61,8 & 1.184 & 79,6 & 742 & 55,2 & 0,001 & 0,001 & 0,001 \\
\hline Centro-Oeste & 163 & 42,2 & 152 & 33,5 & 493 & 53,5 & 0,001 & 0,002 & 0,001 \\
\hline Suldeste & 1.545 & 63,0 & 2.051 & 63,0 & 3.729 & 81,6 & 0,001 & 0,07 & 0,001 \\
\hline Sul & 649 & 47,2 & 512 & 47,4 & 464 & 35,2 & 0,001 & 0,001 & 0,001 \\
\hline
\end{tabular}


Distribuição absoluta e relativa de enfermeiros no Brasil com carga horária semanal de trabalho no emprego principal $\geq 40$ horas segundo os postos de trabalho público, federal, estadual e municipal, entre as regiões geográficas e o ano de pesquisa (PAMS, 2002, 2005, 2009)

\begin{tabular}{|c|c|c|c|c|c|c|c|c|c|}
\hline & 20 & & 20 & & & 09 & p-valor* & p-valor* & p-valor* \\
\hline & $\begin{array}{c}n \\
(25.945)\end{array}$ & $\%$ & $\begin{array}{c}n \\
(38.941)\end{array}$ & $\%$ & $\begin{array}{c}n \\
(55.772)\end{array}$ & $\%$ & $\begin{array}{c}2005 \text { e } \\
2009\end{array}$ & 2002 & 2002 \\
\hline Estadual (Brasil) & 4.646 & 29,4 & 5.226 & 28,3 & 8.629 & 32,8 & 0,001 & 0,001 & 0,001 \\
\hline Norte & 686 & 42,3 & 1.011 & 47,4 & 1.728 & 51,8 & 0,001 & 0,001 & 0,001 \\
\hline Nordeste & 1.318 & 32,6 & 1.641 & 33,4 & 1.843 & 22,8 & 0,001 & 0,001 & 0,001 \\
\hline Centro-Oeste & 601 & 39,3 & 995 & 55,0 & 1.326 & 59,5 & 0,001 & 0.001 & 0,001 \\
\hline Sudeste & 1.430 & 18,3 & 1.052 & 12,2 & 3.296 & 28,5 & 0,001 & 0,001 & 0,001 \\
\hline Sul & 611 & 75,7 & 527 & 53,1 & 436 & 40,0 & 0,001 & 0,001 & 0,001 \\
\hline Municipal (Brasil) & 18.125 & 49,6 & 29.682 & 56,2 & 41.475 & 55,5 & 0,001 & 0,001 & 0,001 \\
\hline Norte & 1.261 & 56,5 & 2.324 & 64,5 & 3.240 & 65,8 & 0,001 & 0,001 & 0,001 \\
\hline Nordeste & 5.933 & 49,1 & 9.278 & 53,0 & 11.703 & 47,4 & 0,001 & 0,001 & 0,001 \\
\hline Centro-Oeste & 1.165 & 60,3 & 1.980 & 66,6 & 2.906 & 68,6 & 0,024 & 0,298 & 0,010 \\
\hline Sudeste & 6.542 & 43,1 & 10.980 & 52,2 & 16.343 & 53,7 & 0,001 & 0,048 & 0,001 \\
\hline Sul & 3.224 & 62,8 & 5.120 & 66,6 & 7.283 & 69,7 & 0,297 & 0,133 & 0,502 \\
\hline
\end{tabular}

Fonte: Instituto Brasileiro de Geografia e Estatística, 2002, 2006, 2010.

*Teste qui-quadrado de Pearson.

Entre as regiões do país, em âmbito federal a proporção de enfermeiros na região Sudeste cresceu significativamente em todos os períodos analisados $(\mathrm{p}<0,001)$; na região Centro-Oeste, cresceu somente entre os dois primeiros anos $(\mathrm{p}<0,002)$, e houve redução no Nordeste e no Sul ( $\mathrm{p}<0,001)$. Em âmbito estadual, as proporções de enfermeiros nas regiões Norte e Centro-Oeste cresceram em todos os períodos analisados ( $p<0,001)$, verificando-se redução relativa nas regiões Sul e Nordeste do país $(\mathrm{p}<0,001)$. Entre os enfermeiros inseridos em âmbito municipal, observou-se maior proporção nas regiões Norte, Centro-Oeste e Sul, e menor no Nordeste e no Sudeste em todos os anos avaliados, mas com aumento significativo ao longo dos anos avaliados apenas para as regiões Norte, Nordeste e Sudeste $(p<0,001)$. Verificou-se redução relativa na região Nordeste do país $(\mathrm{p}<0,001)$ (Tabela 4).

\section{Discussão}

Os resultados do estudo apontaram que no Brasil aumentou a participação de enfermeiros entre os profissionais da saúde inseridos no mercado de trabalho entre os anos de 2002, 2005 e 2009 com carga horária de trabalho $\geq 40$ horas semanais, permanecendo significativos diferenciais nessas proporções em relação a outras profissões de saúde, na distribuição entre as regiões do 
país e no tipo de serviço, público ou privado, ao longo dos anos de estudo da pesquisa.

Estudos prévios mostram que esse perfil de trabalho com carga horária $\geq 40$ horas semanais está associado com a maior participação de enfermeiros no mercado de trabalho, pois aumentou o percentual de enfermeiros entre os profissionais da saúde inseridos no mercado de trabalho entre os anos de 2002, 2005 e 2009, mas permanecem significativos diferenciais geográficos nessa oferta. Embora tenha aumentado a disponibilidade de enfermeiros em algumas regiões e diminuído em outras, a enfermagem ainda é a profissão de saúde de nível superior com maior carga horária semanal no emprego principal (Instituto Brasileiro de Geografia e Estatística, 2002, 2006, 2010); Oliveira, Silva e Carneiro, 2015).

Dois aspectos principais e bastante imbricados podem explicar esse processo de aumento de enfermeiros no mercado de trabalho e com carga semanal $\geq 40$ horas.

O primeiro é a criação e a expansão de instituições e escolas de ensino superior de enfermagem, que determinaram uma explosão de vagas e de egressos de enfermagem disponíveis no mercado de trabalho, havendo um descompasso entre a velocidade de enfermeiros formados e o número de vagas criadas no mercado para os novos profissionais (Oliveira, Silva e Carneiro, 2015). Em contexto de maior competitividade, esse desequilíbrio foi favorável aos empresários do setor privado e gestores do setor público da saúde, que passaram a ter maiores opções de negociação e de barganha para definir salários e carga horária de trabalho mais favorável ao setor patronal.

O segundo relaciona-se à Estratégia Saúde da Família (ESF), que representou importante expansão do mercado de trabalho para os enfermeiros e contribuiu para maior participação dessa categoria no setor de saúde em todo o país. Dados do Ministério da Saúde mostram que em dezembro de 1994 havia 328 equipes de saúde da família; em 2002, aumentou para 16.847; em 2005 já existiam 24.872, e em maio de 2008 o número alcançado foi de 28.302 equipes de saúde da família (Brasil, 2012). Logo, observou-se consistente expansão do número de equipes de saúde da família, com aumento de 40,5\% entre 2002 e 2008. Porém, deve-se considerar que em relação às formas de contratação dos trabalhadores no Sistema Único de Saúde (SUS), somando-se os condicionantes da reforma do Estado na condução da política de recursos humanos para o SUS, essas contratações se caracterizaram pela flexibilização do trabalho e do emprego (Brasil, 2012).

Embora, no Brasil, a resolução do Conselho Federal de Enfermagem (Cofen) n. 293/2004 regulamente escala mensal para pessoal de enfermagem com carga horária semanal de 36 horas para atividades assistenciais e de quarenta horas para atividades administrativas, ainda se observa que a maioria dos enfermeiros tem carga excessiva de trabalho. Essa situação confronta-se com 
a longa luta dos profissionais de enfermagem pela definição de uma jornada de trabalho de trinta horas semanais, a qual já passa por longo período de embates sem ainda alcançar a definição nacional dessa conquista (Pires et al., 2010; Santos et al., 2013; Dalri et al., 2014a).

Esse cenário de discrepância entre o desejado e o real contexto de carga horária de trabalho dos enfermeiros no Brasil estimula o debate sobre a estreita relação dessa carga horária de trabalho com políticas de educação e saúde, fatores socioeconômicos, organizacionais do país e do setor saúde, o que mostra a importância, a relevância e a necessidade de esse tema compor a agenda de pesquisadores no campo da enfermagem (Oliveira, Silva e Carneiro, 2015).

Usualmente, são detectados problemas associados a essa jornada de trabalho, como más condições de trabalho, irregularidade do horário de descanso, quantitativo insuficiente de pessoal, turnos ininterruptos, aumento na jornada diária em consequência da passagem de plantão, além de muita responsabilidade e pouca valorização, comprometendo assim a integridade física e mental do profissional de enfermagem (Pires et al., 2010; Felli, 2012; Trindade e Pires, 2013; Silva Neto et al., 2015). Ressalta-se que o debate sobre a redução e a adequação da jornada de trabalho deve estar articulado à busca por melhoria das condições de trabalho dos profissionais da enfermagem; caso contrário, corre-se o risco de uma conquista estéril (Felli, 2012).

Ao se considerar que os resultados revelam que a enfermagem permanece com carga horária de trabalho excessiva e desfavorável em relação às demais profissões de saúde de nível superior, numa desigualdade que aumentou ao longo dos anos em todas as regiões, observa-se realmente que as demais profissões da saúde já obtiveram conquistas em relação à jornada de trabalho, como no caso dos profissionais médicos, com vinte horas semanais desde 1961; fisioterapeutas e terapeutas ocupacionais, com trinta horas semanais desde 1994; assistentes sociais, com trinta horas semanais desde 2010; e técnicos em radiologia, com 24 horas semanais desde 1985 (Pires et al., 2010; Felli, 2012).

Para a enfermagem, essa conquista vem sendo dificultada por alegações de empregadores do setor privado de saúde e setores do governo de que a redução da carga horária dos profissionais de enfermagem teria enorme impacto financeiro e traria sérios prejuízos econômicos, porque trata-se de uma força de trabalho expressiva, que responde por $65 \%$ da equipe de saúde que atende no sistema. Com registro no conselho de classe em 2015, seria um total de 1,8 milhão de profissionais - destes, 414 mil enfermeiros e 1,4 milhão de técnicos e auxiliares (Pires et al., 2010; Felli, 2012; Santos et al., 2013; Trindade e Pires, 2013; Machado et al., 2016).

Apesar do grande contingente numérico e da influência decisiva do seu trabalho na qualidade das ações em saúde, isto não lhes garante valorização; ao contrário, como trabalho inserido no setor terciário da economia, a enfermagem sofre impactos das políticas econômicas capitalistas do país e, assim, 
não consegue garantir proteção legal ao seu trabalho, com jornada adequada de trabalho regulamentada por lei (Pires et al., 2010; Felli, 2012; Silva Neto et al., 2015; Machado et al., 2016).

Vale ressaltar que o argumento de impacto financeiro decorrente da regulamentação das trinta horas para a categoria merece diversas críticas, pois para a União o impacto seria relativo, uma vez que a maioria dos profissionais do SUS é remunerada por estados e municípios, cabendo a esta esfera apenas os hospitais universitários (HUs). Além disso, muitos servidores da rede pública estadual, municipal, dos HUs e de alguns hospitais privados já adotam as trinta horas. A redução da jornada contribuiria para diminuir o desgaste físico e emocional desses profissionais e, por extensão, permitiria assistência mais segura, redução de ações judiciais contra os serviços de saúde e de gastos com afastamentos por motivos de saúde (Pires et al., 2010; Felli, 2012).

A carga horária $\geq 40$ horas no emprego principal desses profissionais torna-se ainda mais relevante se analisada em associação a outro problema para a enfermagem, que se refere a múltiplos vínculos de trabalho, geralmente estimulados por situações econômicas desfavoráveis e baixa remuneração mantendo tais profissionais com rendimentos mensais aquém do básico para um trabalho decente e com jornadas de trabalho muito elevadas e extenuantes (Pires et al., 2010; Felli, 2012; Machado et al., 2016).

A dupla jornada dos profissionais de enfermagem tem inclusive sido utilizada como argumento para não aprovação das trinta horas, sob a alegação de que tal redução incentivaria os profissionais a buscarem múltiplos vínculos. Nesse sentido, reforça-se a necessidade de se vincular a essa luta a defesa por salários dignos, visando possibilitar subsistência sem a necessidade de extenuante acúmulo de empregos (Pires et al., 2010; Santos et al., 2013).

Ressalta-se ainda, em relação a essa jornada $\geq 40$ horas, que a enfermagem segue como uma profissão exercida em sua maioria por mulheres, que além dessa elevada carga horária e da múltipla jornada enfrentada no âmbito profissional, enfrentam as responsabilidades de dedicação familiar, que lhes é culturalmente atribuída (Pires et al., 2010; Dalri et al., 2014b).

Em relação aos setores público e privado, observaram-se elevadas proporções e com aumento ao longo dos anos, com importantes diferenças entre as regiões do país, ressaltando-se que o setor público abrigou grande parte dos enfermeiros com carga horária de trabalho semanal $\geq 40$ horas no Brasil em todos os anos avaliados. Esse padrão de distribuição pode estar relacionado ainda com o fato de que no Brasil, ao longo dos últimos anos, a maioria dos enfermeiros atua em serviços públicos de saúde, e o padrão de distribuição observado no setor privado indica possível estabilização ou menor participação relativa desse setor como atual campo de trabalho para a enfermagem brasileira (Oliveira, Silva e Carneiro, 2015). 
A pesquisa "Perfil da enfermagem no Brasil" mostrou que o setor público possui mais de 75.000 estabelecimentos de saúde e gera cerca de 270 mil vagas para enfermeiros, abrigando assim mais de $65 \%$ do quadro desses profissionais (Machado et al., 2016). Assim, a maior proporção de enfermeiros com carga horária de trabalho semanal $\geq 40$ horas inseridos em serviços de níveis federal e municipal pode estar relacionada tanto com a maior oferta de serviços de saúde em âmbito público como com a falta de regulamentação da jornada de trabalho em âmbito nacional.

Os resultados sobre o setor privado apontaram maior proporção de enfermeiros com carga horária de trabalho semanal $\geq 40$ horas no emprego principal nas regiões Centro-Oeste, Sudeste e Sul. Tal achado pode estar relacionado à maior capacidade instalada de serviços de saúde nessas regiões que são socioeconomicamente as mais desenvolvidas do país. Na prática, a jornada ideal de trabalho da enfermagem não está regulamentada em lei, valendo a livre negociação, que varia entre trinta horas semanais, já adotada em alguns espaços do setor público e privado, e de 36, 40 ou 44 horas praticadas pela maioria dos serviços (Santos et al., 2013; Silva Neto et al., 2015; Machado et al., 2016).

Entre as condições laborais dos profissionais de enfermagem no Brasil, a jornada de trabalho tem representado a principal questão de debate e preocupação nacional, pois se constitui em um dos vários tipos de estressores relacionados ao trabalho, caracterizando-se como um grande problema ocupacional e um dos principais desafios da enfermagem moderna (Gray-Stanley e Muramatsu, 2011; Bogaert et al., 2013; Lima et al., 2013). Esse debate busca promover o controle da carga horária de trabalho semanal e instituir um padrão desejável e adequado de condições de jornada de trabalho no país que levem a melhor resultado assistencial, qualidade dos serviços e maior qualidade de vida laboral (Pires et al., 2010).

Nos últimos anos, o panorama evolutivo da distribuição dos enfermeiros nos serviços públicos e privados de saúde observados no Brasil tem refletido a tendência de municipalização da gestão da atenção à saúde - e a menor atuação das esferas federal e estadual na execução de serviços públicos desse setor. Logo, muitos municípios e estados vêm instituindo jornada de trabalho inferior a quarenta horas semanais. No entanto, em âmbito federal, principalmente nas estratégias de capilarização da saúde financiadas pelo governo federal, como por exemplo a ESF, a carga horária tem sido mantida em quarenta horas semanais (Pires et al., 2010; Dalri et al., 2014a).

Dessa forma, apesar das amplas vantagens associadas à redução da carga horária de trabalho semanal, estudos mostram que os desafios para seu alcance ainda são grandes e complexos, sobretudo em um país de dimensões continentais e diversidades de tipos de serviços de saúde como os encontrados no Brasil (Pires et al., 2010; Dalri et al., 2014a, 2014b). 
Apesar dos resultados obtidos no estudo aqui apresentado, convém destacar algumas limitações relacionadas a eles, como a possibilidade de divergência entre o quantitativo de enfermeiros regularmente registrados no Cofen e aqueles que formaram o conjunto de enfermeiros inseridos no mercado de trabalho, o que pode estar associado ao fato de que existem enfermeiros com registro no conselho, mas que trabalham em funções não relacionadas a assistência, saúde ou enfermagem - bem como aqueles sem ocupação e sem qualquer posição no mercado de trabalho, os quais por isso não foram identificados como enfermeiros durante a realização da PAMS. Também é possível que o mesmo profissional esteja registrado em mais de uma instituição ou serviço de saúde da mesma região ou de diferentes regiões, o que pode indicar a dupla sobrecarga de trabalho em razão do multiemprego e da ocupação de mais de um posto de trabalho por um mesmo profissional. No entanto, essa questão não deve limitar a reflexão sobre os problemas associados à desigual sobrecarga de trabalho de enfermeiros no Brasil, pois os dados utilizados na pesquisa foram obtidos por meio de censo de amplitude nacional, que permite obter estimativas com boa representatividade da realidade brasileira. Ademais, os resultados indicam a real oferta de enfermeiros no início do século XXI inseridos no cotidiano do mercado de trabalho que exercem carga horária de trabalho maior ou igual a quarenta horas semanais no principal emprego.

\section{Conclusões}

A enfermagem é um campo de conhecimento específico e uma prática social que se consolida e se fortalece como ciência, tecnologia e inovação. Apresenta importante contribuição para o conhecimento social, qualidade de vida, tecnologia e inovação em saúde. Entretanto, os resultados indicam que a jornada de trabalho $\geq 40$ horas semanais no Brasil consiste em desafio para os enfermeiros e para a enfermagem como profissão, demonstrando assim que, para o alcance das desejadas trinta horas semanais, faz-se urgente a necessidade de enfrentamento dos artifícios de mercado operados por instituições públicas e privadas que mantêm a enfermagem e os seus profissionais com jornada de trabalho excessiva, desgastante e pouco remunerada.

A enfermagem ainda é uma profissão em que a formação de novos profissionais, a distribuição deles no mercado de trabalho e a carga horária de trabalho semanal são mais explicadas por fatores socioeconômicos e políticos do que por elementos relacionados à organização e ao planejamento do setor saúde e à necessidade de bem-estar e qualidade de vida laboral e da população em geral. Logo, a definição legal de uma jornada de trinta horas poderá fortalecer a enfermagem como campo de conhecimento específico e como prática social que se consolida como ciência, tecnologia e inovação. 
A nova jornada de trabalho pode resultar em ganhos sociais e prestação de cuidados seguros e de qualidade. Assim, a enfermagem tem relevante responsabilidade na assistência em saúde, o que requer condições de trabalho e conhecimentos científicos adequados para uma prática orientada por ideais de justiça social e direito à vida.

\section{Colaboradores}

Bruno Luciano Carneiro Alves de Oliveira, Alécia Maria da Silva e Sara Fiterman Lima participaram igualmente de todas as fases de elaboração do manuscrito e aprovaram a versão final do artigo.

\section{Agradecimento}

Os autores agradecem à Fundação de Amparo à Pesquisa e ao Desenvolvimento Científico e Tecnológico do Maranhão (Fapema) pelo auxílio recebido por meio do Edital Apoio à Publicação de Artigos Científicos.

Resumen Este estudio tuvo como objetivo presentar la distribución de enfermeros en el mercado de trabajo de Brasil con carga horaria semanal mayor o igual a cuarenta horas, según la región y el tipo de lugar de trabajo, es decir, público o privado. Se trató de un estudio transversal, realizado entre el 10 y el 27 de febrero del 2017, con datos on-line de las investigaciones de asistencia médicosanitaria realizadas en el 2002, 2005 y 2009 por el Instituto Brasileño de Geografía y Estadística. Se analizó un total de 179.337 enfermeros en los tres años evaluados. Se compararon sus proporciones de acuerdo con las regiones geográficas y el lugar de trabajo utilizando la prueba de Chi-cuadrado de Pearson $(a=5 \%)$. La enfermería fue la única de las profesiones de la salud de nivel superior que presentó una creciente y mayor proporción de profesionales con carga de trabajo mayor o igual a cuarenta horas semanales $(\mathrm{p}<0,001)$ en los años evaluados, siendo la mayor proporción en el sector público y en las regiones Norte, Centro-Oeste y Sur del país. La enfermería aún permanece con una carga de trabajo desfavorable, lo que representa un desafío para los enfermeros y para la enfermería como profesión.

Palabras clave enfermería; salud y desigualdad; mercado de trabajo. 


\title{
Notas
}

\author{
${ }^{1}$ Universidade Federal do Maranhão, Pinheiro, Maranhão, Brasil. \\ <brunodeoliveirama@gmail.com> \\ Correspondência: Universidade Federal do Maranhão, Estrada Pinheiro, Pacas, km 10, \\ s/n, Enseada, CEP 65200-000, Pinheiro, Maranhão, Brasil. \\ ${ }^{2}$ Prefeitura de Cururupu, Secretaria Municipal de Saúde, Cururupu, Maranhão, Brasil. \\ $<$ alemapb81@gmail.com> \\ ${ }^{3}$ Universidade Federal do Maranhão, Programa de Pós-Graduação em Saúde Coletiva, \\ Pinheiro, Maranhão, Brasil. \\ <sara.fiterman@gmail.com>
}

\section{Referências}

BOGAERT, Peter V. et al. The relationship between nurse practice environment, nurse work characteristics, burnout and job outcome and quality of nursing care: a cross-sectional survey. International Journal Nurse Studies, Amsterdam, v. 2.231, p. 1-11, 2013. Disponível em: <https://www.ncbi.nlm.nih.gov/ pubmed/23777786> . Acesso em: 17 jun. 2017.

BRASIL. Ministério da Saúde. Secretaria de Gestão Estratégica e Participativa. Departamento de Monitoramento e Avaliação da Gestão do SUS. Painel de indicadores do SUS n. 4: saúde da família. Brasília, DF: Ministério da Saúde, 2012. Disponível em: <http:// bvsms.saude.gov.br/bvs/publicacoes/tematico_saude_familia_v_2_n_4.pdf $>$. Acesso em: 25 mai. 2017.

DALRI, Rita C. M. B. et al. Carga horária de trabalho dos enfermeiros e sua relação com as reações fisiológicas do estresse. Revista Latino-Americana de Enfermagem, Ribeirão Preto, v. 22, n. 6, p. 959-965, nov./dez. 2014a. Disponível em: <http://www.scielo.br/pdf/ rlae/v22n6/pt_0104-1169-rlae-22-06-00959. pdf>. Acesso em: 22 jul. 2017.

DALRI, Rita C. M. B. et al. Carga horária dos enfermeiros de emergência e sua relação com estresse e cortisol salivar. Revista de Enfermagem UFPE (online), Recife, v. 8, n. 10, p. 3.358-3.368, 2014b. Disponível em: <https:// periodicos.ufpe.br/revistas/revistaenfermagem/ article/view/10067/0 > . Acesso em: 15 fev. 2016.

FELLI, Vanda E. A. Condições de trabalho de enfermagem e adoecimento: motivos para a redução da jornada de trabalho para 30 horas. Enfermagem em Foco, Brasília, v. 3, n. 4, p. 178-181, 2012. Disponível em: <http://revista. portalcofen.gov.br/index.php/enfermagem/ article/view/379 > . Acesso em: 26 jan. 2016.

GRAY-STANLEY, Jennifer A.; MURAMATSU, Naoko. Work stress, burnout, and social and personal resources among direct care workers. Research in Developmental Disabilities, Chicago, v. 32, n. 3, p. 1.065-1.074, 2011. Disponível em: <https://www.ncbi.nlm.nih.gov/pubmed/21316918>. Acesso em: 25 mar. 2016.

INSTITUTO BRASILEIRO DE GEOGRAFIA E ESTATÍSTICA (IBGE). Departamento de População e Indicadores Sociais. Estatísticas de saúde. Assistência Médico-Sanitária 2002. Rio de Janeiro: IBGE, 2002. Disponível em: <https://www.ibge.gov.br/estatisticas-novoportal/ sociais/saude/9067-pesquisa-de-assistenciamedico-sanitaria.html?edicao $=17212 \& \mathrm{t}=$ sobre $>$. Acesso em: 25 nov. 2015.

INSTITUTO BRASILEIRO DE GEOGRAFIA E ESTATÍSTICA (IBGE). Departamento de População e Indicadores Sociais. Coordenação de População e Indicadores Sociais. Estatísticas 
de Saúde. Assistência Médico-Sanitária 2005. Rio de Janeiro: IBGE, 2006. Disponível em: < https://www.ibge.gov.br/estatisticas-novoportal/ sociais/saude/9067-pesquisa-de-assistenciamedico-sanitaria.html? edicao $=17211 \& \mathrm{t}=$ sobre $>$. Acesso em: 25 nov. 2015.

INSTITUTO BRASILEIRO DE GEOGRAFIA E ESTATÍSTICA (IBGE). Departamento de População e Indicadores Sociais. Coordenação de População e Indicadores Sociais. Estatísticas de Saúde. Assistência Médico-Sanitária 2009. Rio de Janeiro: IBGE, 2010. Disponível em: < https://www.ibge.gov.br/estatisticas-novoportal/ sociais/saude/9067-pesquisa-de-assistenciamedico-sanitaria.html?edicao $=9275 \& \mathrm{t}=$ sobre $>$. Acesso em: 25 nov. 2015.

LALA, Adrian I. et al. Coping behavior and risk and resilience stress factors in French regional emergency medicine unit workers: a cross-sectional survey. Journal Medical and Life, Amsterdam, v. 9, n. 4, p. 363-368, out.dez. 2016. Disponível em: <https://www. ncbi.nlm.nih.gov/pmc/articles/PMC5141395/>. Acesso em: 9 set. 2017.

LIMA, Marlinir B. et al. Agentes estressores em trabalhadores de enfermagem com dupla ou mais jornada de trabalho. Revista de Pesquisa Cuidado é Fundamental (on-line), Rio de Janeiro, p. 3.259-3.266, 2013. Disponível em: $<$ http://biblioteca.cofen.gov.br/wp-content/ uploads/2015/02/agentes-estressores-emtrabalhadores-de-enfermagem-com-duplaou-mais-jornadas-de-trabalho.pdf $>$. Acesso em: 9 out. 2017.

MACHADO, Maria H. et al. Mercado de trabalho da enfermagem: aspectos gerais. Enfermagem em Foco, Brasília, v. 7, n. esp., p. 35-53, 2016. Disponível em: <http://revista.portalcofen.gov. br/index.php/enfermagem/article/view/69l>. Acesso em: 11 out. 2017.
OLIVEIRA, Bruno L. C. A.; SILVA, Alécia M.; CARNEIRO, Alan D. A distribuição de enfermeiros no Brasil segundo as pesquisas de assistência médico-sanitária (2002, 2005, 2009). Revista Eletrônica Gestão \& Saúde. Brasília, v. 6, n. 2, p. 1.334-1.353, 2015. Disponível em: $<$ http://periodicos.unb.br/index.php/rgs/article/ view/22471/16068> . Acesso em: 22 ago. 2016.

PIRES, Denise et al. Jornada de 30 horas semanais: condição necessária para assistência de enfermagem segura e de qualidade. Enfermagem em Foco, Brasília, v. 1, n. 3, p. 114-118, 2010. Disponível em: <http://revista.portalcofen.gov.br/index.php/enfermagem/article/ viewFile/182/119>. Acesso em: 22 ago. 2016.

SANTOS, Tatiane A. et al. Significado da regulamentação da jornada de trabalho em enfermagem. Revista Enfermagem Uerj, Rio de Janeiro, v. 21, n. 2, p. 265-268, 2013. Disponível em: <http://www.e-publicacoes.uerj.br/ index.php/enfermagemuerj/article/view/7224>. Acesso em: 27 set. 2016.

SILVA NETO, José A. et al. Aspectos jurídicos da jornada de trabalho em enfermagem: reflexão teórica. Revista de Enfermagem da Universidade Federal do Piauí, Teresina, v. 4, n. 3, p. 95-98, jul.-set. 2015. Disponível em: <http://www. ojs.ufpi.br/index.php/reufpi/article/view/2368/ pdf>. Acesso em: 27 set. 2016.

TRINDADE, Letícia L.; PIRES, Denise E. P. Implicações dos modelos assistenciais da atenção básica nas cargas de trabalho dos profissionais de saúde. Texto \& Contexto Enfermagem, Florianópolis, v. 22, n. 1, p. 36-42, 2013. Disponível em: <http://www.scielo.br/pdf/tce/ v22nl/pt_05.pdf>. Acesso em:15 ago. 2016.

Recebido em 26/11/2017.

Aprovado em 23/03/2018. 